\title{
Influence of the Local Morphology on the Surface Tension of Injection Molded Polypropylene
}

\author{
M. Gomes*, A.J. Pontes and J.C. Viana \\ IPC/I3N - Institute for Polymers and Composites/Institute of Nanostructures, Nanomodelling and Nanofabrication \\ Department of Polymer Engineering, University of Minho, Portugal-mgomes@dep.uminho.pt; \\ pontes@dep.uminho.pt; jcv@dep.uminho.pt
}

\begin{abstract}
In this work, we investigate the development of the morphology of an injection molding polypropylene under the local thermomechanical environment imposed during processing, and its effect on the contact angle and, hence, on the surface tension of the moldings. Melt and mold temperatures were varied in two levels. The local thermomechanical environment was characterized by mold filling computational simulations that allow the calculation of thermomechanical variables (e.g., local temperatures, shear stresses) and indices (related to the local morphology development). In order to investigate the structural hierarchy variations of the moldings in the thickness direction, samples from skin to core were used. The molecular orientation and degree of crystallinity were determined as function of the thickness, as well as the contact angle. The variations of the degree of crystallinity were assessed by differential scanning calorimetry. The level of molecular orientation was evaluated by birefringence measurements. The contact angles were measured in deionized water by sessile drop (needle in) method at room temperature, to determine the wettability of the samples. The contact angles were found to vary along the molding thickness in the skin, transition and core layers. These variations are related to the local morphologies developed. Results suggest that water contact angle increases with the level of molecular orientation and for finer microstructures.
\end{abstract}

Keywords: Morphology, contact angle, injection molding, crystallinity, molecular orientation

\section{INTRODUCTION}

Injection overmolding is a well-established process to manufacture multi-component plastic products. The interaction between the different materials to be molded strongly depends on the type of materials and on the functional groups present. Obviously, adhesion plays a relevant role in multi-component parts. Processing conditions play also a relevant role in adhesion as they constrain the development of the interphase morphology. For example, it has been reported that crystallinity of a semicrystalline polymer has an effect on its adhesive properties $[1,2]$. It is also expected that flow conditions should have an influence on the interphase morphology and therefore in the adhesion. Processing conditions may also promote poor adhesion due to the creation of residual stresses at the interface. Furthermore, the manipulation of interface/interphase morphology during processing is also important.

\section{Microstructure Development}

The microstructure developed by semicrystalline thermoplastics when cooled down under specific thermomechanical conditions, namely shear fields and important thermal gradients, is widely reported in the literature $[3,4]$.

Generally, a 3-layered structure featuring a hierarchy of macromolecular arrangements and a through-thethickness morphological gradient, consisting of an oriented skin layer, a transition layer (shear-induced structure) and a highly crystalline core can be observed in polarized light. The number of identified layers depends on the level of discrimination considered and on the degree of resolution of the experimental technique used $[5,6]$.

The skin layers start forming during the filling phase due to the rapid cooling of the hot and very oriented melt against to the cold mold walls (development of the frozen layer). When observed by polarized light microscopy, the skin has a homogeneous appearance with no discernible morphological features. However, it has a peculiar microstructure, characteristic of crystallization under high stress fields and cooling rates, normally referred to as shish-kebab structures [4, 7 and 8]. The skin thickness is controlled by the combined effect of the cooling rate and of the stress fields imposed to the melt. Its formation is thermally controlled for higher melt temperatures and governed by the shear stress level for the lowest melt temperatures $[9,10]$. Thicker skins are promoted by lower molding temperatures (melt and mould) and flow rates and by higher pressures [9-13].

The core zone shows essentially an $\alpha$-type spherulitic morphology, typical of melts that crystallize in almost quiescent conditions. The previously crystallized material acts as an insulation barrier due to its low thermal conductivity, leading to a slower cooling rate in the core, which is minimum at the core center. Consequently, the core has generally a high crystallinity, showing a spherulite size gradient (typically of the order of $1-10 \mu \mathrm{m}$ of diameter) with dimensions increasing towards the central zone. The slower crystallization process in the core is mainly thermally controlled. The final degree of crystallization 
of the core decreases with increasing cooling rate and for low melt temperatures $[12,14]$.

The evolution from the skin to core is progressive, and sometimes a transition zone can be defined in between (also called shear zone). The morphology and thickness of this zone are mainly dependent on the holding stage conditions of the molding cycle. The degree of crystallinity in the transition zone is noticeably affected by the holding pressure. An increase on it acts as a quenching phenomenon (the pressure increases the thermodynamic melting temperature originating a higher undercooling degree), which can locally reduce the degree of crystallinity [15].

The molecular orientation is defined as the preferred alignment of the molecular chains in given direction. For semicrystalline materials, this is directly related to the factors affecting the relaxation and crystallization processes, that is the morphology development. Due to the imposed boundary cooling conditions, the molecular orientation profile in the thickness direction is normally symmetrical relatively to the middle plane, unless for the cases of unbalance cooling [29] and of flow disturbances (e.g., abrupt thickness variations).

\section{Thermodynamics of adhesion}

The equilibrium contact angle, $\theta_{0}$, results from the balancing of surface tension forces along the line of intersection for the contacting phases. This is described by the well-known Young's equation (Eq.1), that is:

$$
\gamma_{1 \mathrm{v}} \cos \theta_{0}+\gamma_{\mathrm{sl}}=\gamma_{\mathrm{sv}}
$$

in which $\gamma_{\mathrm{ij}}$ is the tension for interfaces formed between solid (s), liquid (1), and vapor (v) phases.

Contact angle measurements have been used for many years to assess surface. The contact angle is defined as the angle between the tangent to the liquidair interface and the tangent to the solid-air interface, as shown in Fig. 1. Contact angles less than $90^{\circ}$ correspond to hydrophilic surfaces, while hydrophobic surfaces are defined by larger contact angles. The contact angle on an ideal smooth surface is known as Young's contact angle.

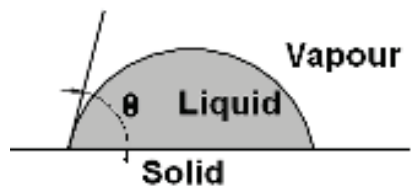

Fig. 1 - A schematic illustration of the contact angle between a liquid drop onto a solid surface.

Neglecting the effects of gravity, the Young angle can be explicitly related to solid-vapor $\left(\gamma_{\mathrm{SV}}\right)$, solid-liquid $\left(\gamma_{\mathrm{SL}}\right)$, and liquid-vapor $\left(\gamma_{\mathrm{LV}}\right)$ interfacial surface energies:

$$
\cos \theta_{Y}=\frac{\gamma_{S V}-\gamma_{S L}}{\gamma_{L V}}
$$

Therefore, contact angles are influenced by the specific kinds of atoms and surface terminations present at the liquid-solid-vapor interfaces.

In this work, we investigate the development of the microstructure of an injection molding polypropylene under the local thermomechanical environment imposed during processing, and its effect in the contact angle and, hence, on adhesion.

\section{EXPERIMENTAL}

\section{Materials and Processing Conditions}

A commercial unfilled homopolymer polypropylene Moplen HP648T, from Lyondell Basell Industries, was selected to produce the injection molding samples (Fig. 2) using a 200Ton Billion Hercule 200 injection molding machine. Two processing parameters were varied in this study; the mold temperature $\left(\mathrm{T}_{\mathrm{w}}\right)$ and the melt temperature $\left(\mathrm{T}_{\mathrm{m}}\right)$, in order to obtain different morphologies. The mold temperature was varied between 25 and $60^{\circ} \mathrm{C}$, and the melt temperature varied between 210 and $250^{\circ} \mathrm{C}$ (Table 1). Other parameters, like injection speed $\left(\mathrm{V}_{\text {inj }}\right)$, holding pressure $\left(\mathrm{P}_{2}\right)$ and holding time $\left(\mathrm{t}_{2}\right)$ here kept constant.

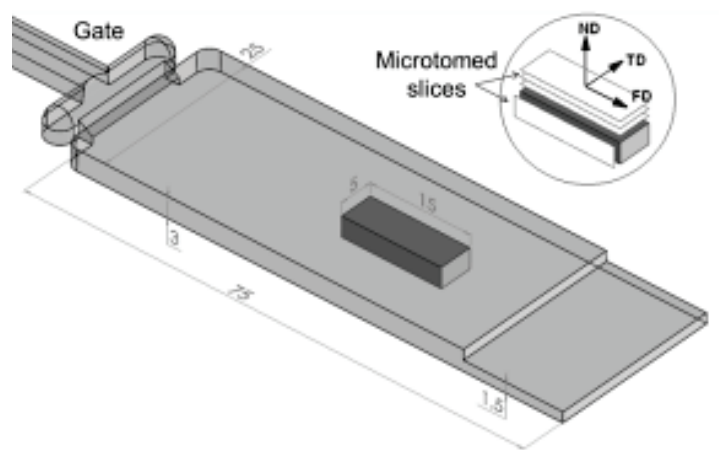

Fig. 2 - Injection molding samples.

\begin{tabular}{|c|cc|ccc|}
\multicolumn{7}{c|}{ Table 1- Molding conditions. } \\
\cline { 2 - 5 } \multicolumn{1}{c|}{} & $\begin{array}{c}\mathrm{T}_{\mathrm{m}} \\
\left({ }^{\circ} \mathrm{C}\right)\end{array}$ & $\begin{array}{c}\mathrm{T}_{\mathrm{w}} \\
\left({ }^{\circ} \mathrm{C}\right)\end{array}$ & $\begin{array}{c}\mathrm{V}_{\mathrm{inj}} \\
(\mathrm{mm} / \mathrm{s})\end{array}$ & $\begin{array}{c}\mathrm{P}_{2} \\
(\mathrm{bar})\end{array}$ & $\begin{array}{c}\mathrm{t}_{2} \\
(\mathrm{~s})\end{array}$ \\
\hline $\mathrm{C}_{1}$ & 210 & 25 & \multirow{2}{*}{10} & 200 & 1.5 \\
\hline $\mathrm{C}_{2}$ & 250 & 60 & & & \\
\hline
\end{tabular}

\section{Sample Preparation}

The studied zone was located $45 \mathrm{~mm}$ from the gate, at the center of the part. Samples of $15 \mathrm{~mm}$ length and $5 \mathrm{~mm}$ width were cut from the injection molded parts for each condition, as depicted in Fig. 2. In each sample, a slice normal to the flow direction (FD) in the FD-normal direction (ND) was microtome, using an Anglia Scientific microtome. These specimens were then immersed in Canada balsam resin (with the same reflection index of the glass) between two microscopic glasses slides. These sections were used to determine, by polarized light microscopy, the thickness of the skin layer (Figure 3). The micrographs were obtained using 
a Polarized Light microscope Olympus BH-2 UMA with a digital Leica camera.

Then, slices of $10 \mu \mathrm{m}$ were microtome parallel to the flow direction (FD) in the FD-transverse direction (TD) plane, at a distance of $60 \mu \mathrm{m}$ apart from each other (Figure 2).
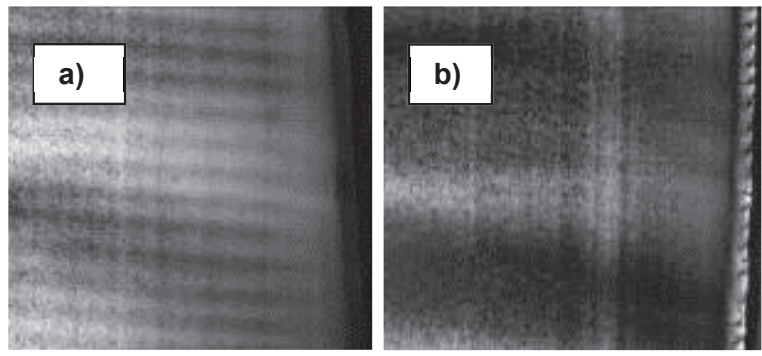

Fig. 3 - Section, normal to the flow direction for conditions a) $\mathrm{C} 1$ and b) $\mathrm{C} 2$.

\section{Microstructure Characterization}

In order to investigate the structural hierarchy variations in the thickness direction, samples from skin to core layered on the FD-TD plane (Figure 2) were used.

\section{- $\quad$ Degree of Crystallinity}

Calorimetric studies were performed in order to assess the variations of the degree of crystallinity along the thickness of the sample. The DSC measurements were performed in a PERKIN ELMER DSC-7 series differential scanning calorimeter, at heating rate of $10^{\circ} \mathrm{C} / \mathrm{min}$ in a nitrogen atmosphere, and from 40 to $200^{\circ} \mathrm{C}$. The equipment was calibrated with pure indium, In. The degree of crystallinity $\left(\chi_{c}\right)$ was calculated by Eq. 3:

$$
X_{c}=\frac{\Delta \mathrm{H}}{\Delta \mathrm{H}_{0}} \times 100
$$

where $\Delta H$ is the enthalpy of fusion of the sample and $\Delta \mathrm{H}_{0}$ is the theoretical enthalpy of fusion for a $100 \%$ crystalline PP. For each scan, $\Delta \mathrm{H}$ was obtained by integration of the DSC curve between 140 and $170^{\circ} \mathrm{C}$. The mass crystallinity values reported are averaged results from at least three identical samples and were calculated using a $\Delta \mathrm{H}_{0}$ of $148 \mathrm{~J} / \mathrm{g}$ [16].

\section{- $\quad$ Birefringence Measurements}

The orientation degree was accessed by birefringence measurements on the samples cut.

A Berek compensator was employed to measure the birefringence of the specimens with a Polarized Light microscope Olympus BH-2 UMA.

\section{- $\quad$ Dynamic Contact Angle Measurements}

Contact angles were measured with deionized water on a Dataphysics OCA15 plus instrument by sessile drop (needle in) method at room temperature to analyze the wettability of the samples.
A drop of the deionized water with a volume of $3 \mu \mathrm{L}$ was delivered to the insert surface by a microsyringe pump. The drop volume was selected so that there is no interference from gravity. The procedure was repeated seven times for each sample giving a standard error for the mean value of approximately $5^{\circ}$. Preliminary surface cleaning was performed using isopropyl alcohol at room temperature. Subsequently, specimens were rinsed with distilled water.

\section{RESULTS AND DISCUSSION}

\section{Microstructure Characterization}

- Degree of Crystallinity

Fig. 4 shows the variation of the degree of crystallinity through the thickness of the sample with the superimposition of a microscopic view under polarized light of the samples cut in the FD-ND direction. As expected, the degree of crystallinity is higher and constant at the core of the sample, despite a peak in the transition zone (condition $\mathrm{C} 1$ ), which has been explained before $[17,18]$ by the combined effect of the flow-induced crystallization and of the pressure on the crystallization temperature.

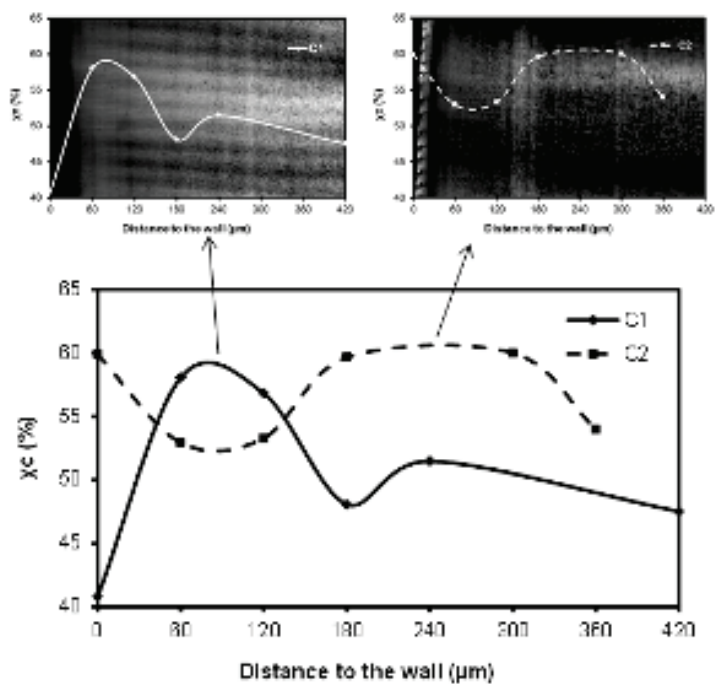

Fig. 4 - Degree of crystallinity variation within the thickness of the specimen. Microscopic view under polarized light superimposition, for each condition, $\mathrm{C} 1$ and $\mathrm{C} 2$.

- $\quad$ Birefringence Measurements

The variation of the molecular orientation, accessed by birefringence measurements, through the thickness of the samples is shown in Fig. 5.

As expected the molecular orientation is higher at the center of the skin layer. However a second peak is observed for both samples at the core layer, which is due to the holding pressure. 


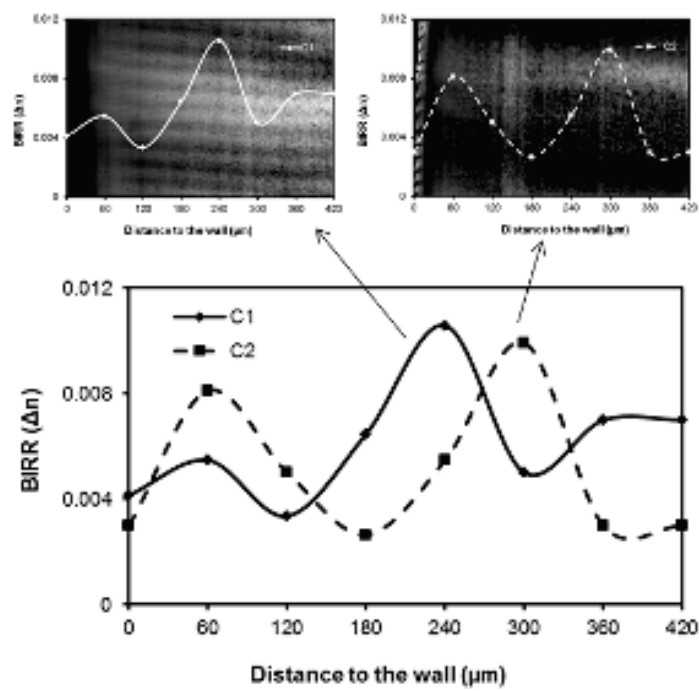

Fig. 5 - Molecular orientation variation within the thickness of the specimen. Microscopic view under polarized light superimposition, for each condition, $\mathrm{C} 1$ and $\mathrm{C} 2$.

\section{- Dynamic Contact Angle Measurements}

In Table 2 are summarized the contact angle measurements for each layer, with the values of the degree of crystallinity and molecular orientation for the same layers.

Table 2 - Contact angle measurements for each layer.

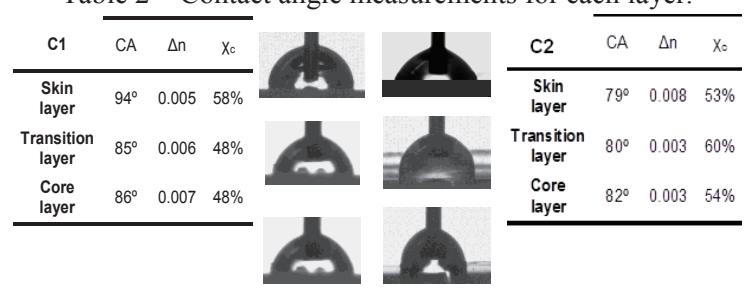

The impact that the degree of crystallinity and the molecular orientation exert in the contact angle of the sample, respectively, is depicted in Fig. 6 and Fig. 7.

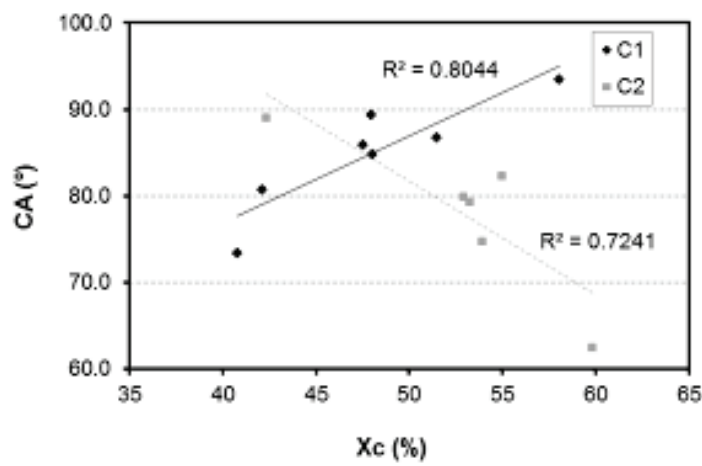

Fig. 6 - Influence of degree of crystallinity in the contact angle.

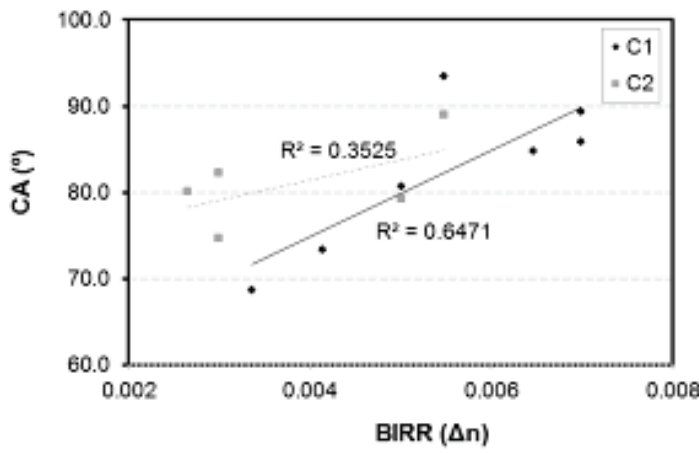

Fig. 7 - Influence of molecular orientation in the contact angle.

The degree of crystallinity appears to have a contradictory influence in the contact angle of the sample. For condition $\mathrm{C} 1$ (rapid cooling), the contact angle increases with degree of crystallinity, but it decreases with it for faster cooling (condition C2).

In the case of molecular orientation, it is shown that the higher the orientation of the chains, the higher the water contact angle.

\section{Microstructure - Contact Angle Relationship}

The relationship between the microstructure of the sample (degree of crystallinity and molecular orientation) and its water contact angle for each condition is depicted in Fig. 8.

Once again, the molecular orientation seems to exert a considerable influence in water contact angle for both conditions, with contact angle increasing with molecular orientation. Unlike molecular orientation, the degree of crystallinity influence in contact angle is moderate, especially for slower cooling (C2).

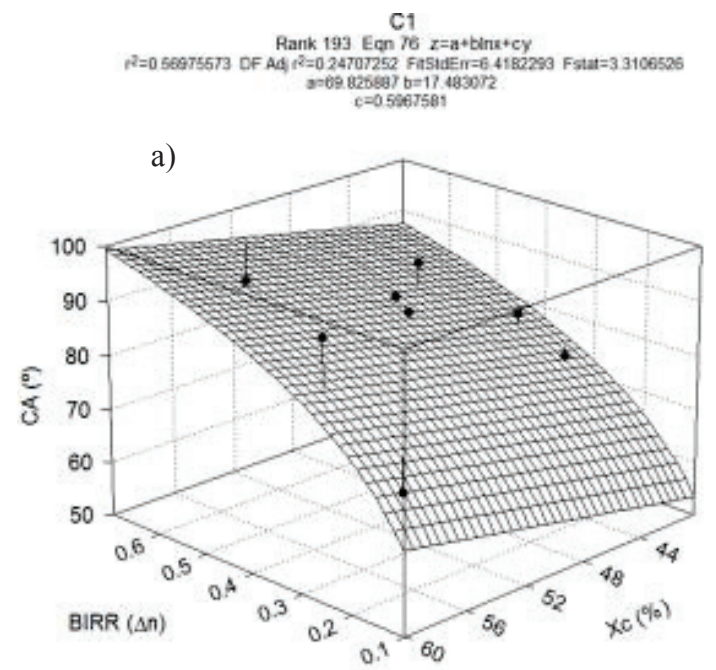




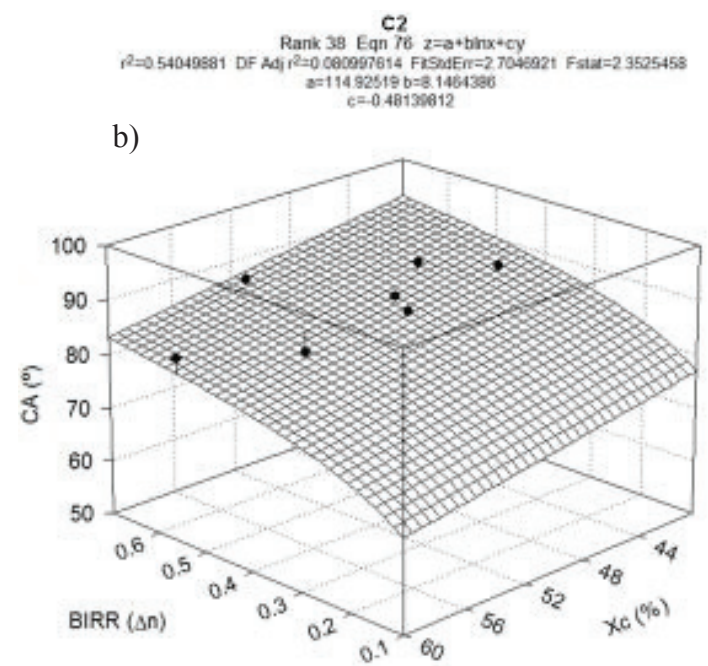

Fig. 8 - Interaction between dynamic contact angle, CA, crystallinity degree, $\chi_{c}$, and orientation, BIRR for conditions a) $\mathrm{C} 1$ and b) $\mathrm{C} 2$.

This fact becomes even more pronounced when expanding the results for the combination of both conditions (Fig. 9). The explanation for this fact may be related to the topography of the sample, i.e. with the crystallite size, as bigger crystallites may have the same effect as micro roughness.

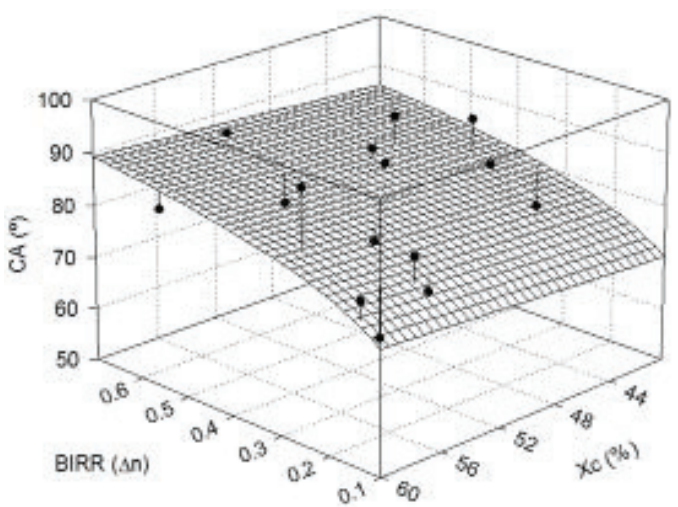

Fig. 9 - Interaction between dynamic contact angle, CA, crystallinity degree, $\chi_{c}$, and orientation, BIRR, for both conditions, $\mathrm{C} 1$ and $\mathrm{C} 2$ (The 3D plane is a fit of the data $\mathrm{CA}=82.32+8.15 \ln \chi \mathrm{c}+0.17 \Delta \mathrm{n} ; \mathrm{R} 2=0.29)$.

\section{CONCLUSION}

It is demonstrated that the morphology developed in the sample due to the processing conditions have an influence in its contact angle.

The molecular orientation is shown to influence greatly the water contact angle, as it increases with increasing orientation of the chains.
The degree of crystallinity also appears to have an influence on contact angle although milder, and even contradictory, depending on the cooling rate, evidencing that it is probably the size of spherulite that is influencing the water contact angle rather than degree of crystallinity. The variation of the water contact angle in this case is probably related to the topography of the sample, as it function like micro roughness in the topography of the surface of the sample. This fact also explains the influence of molecular orientation in contact angle.

\section{REFERENCES}

1. J. Duchet, J.-F. Gérard, J.-P. Chapel, B. Chabert, and J. Brisson, J. Appl. Pol. Sci., 2003, 87, 214

2. E. Boucher, J. P. Folkers, H. Hervet, L. Léger, and C. Creton, Macromolecules, 1996, 29, 774

3. A. Woodward, Understanding Polymer Morphology, Hanser Publisher, Munch, 1995.

4. M. Fujiyama, T. Wakino, Y. Kawasaki, J. App. Pol. Sci., $1988,35,29$

5. B. A. G. Schrauwen, et al, Macromolecules, 2004, 37, 8618

6. Z. Mencik, D. R. Fitchmun, Pol. Sci., 1973, 11, 973

7. G. Kalay, M. J. Bevis, J. Pol. Sci: Part B: Pol. Phys., 1997, 35, 265

8. W. Wening, F. J. Herzog, J. Appl. Pol. Sci., 1993, 50, 2163

9. A. M. Brito, A. M. Cunha, A. S. Pouzada, R. J. Crawford, Int. Pol. Proc., 1991, 4, 370

10. J. C. Viana, A. M. Cunha, N. Billion, Int. Pol, 1997, 43, 159

11. S. S. Katti, J. M. Schultz, Pol. Eng. Sci., 1982, 22, 1001

12. M. Fujiyama In: Polypropylene structure blends and composites: structure and morphology, Karger-Koscis J. editor, Chapman \& Hall, London, 1995, Vol. 1, 167-204

13. M. Demiray, A. I. Isayev, SPE ANTEC Technical Papers, 1996

14. B. Sanschagrin, N. Amdouini, B. Fisa, SPE ANTEC Technical Papers, 1991

15. J. P. Trotignon, J. Verdu, J. Appl. Pol. Sci. 1987, 34, 1

16. B. Monasse, J.-M. Haudin, Colloyd \& Pol. Sci., 1985, 263, 822

17. M. R. Kamal and A. I. Isayev, Injection Molding: Technology and Fundamentals. Carl Hanser GmbH, 2009.

18. T. A. Osswald, L. S. Turng, and P. J. Gramann, Injection Molding Handbook. Hanser, 2008. 